\title{
Caracterización anatómica del árbol bronquial y la división lobular del pulmón del perro
}

\author{
Anatomic characterization of bronchial tree and lobular division \\ of the dog lung
}

\section{Caracterização anatómica da árvore bronquial e divisão lobular do pulmão do cão}

\author{
Elena Blanco - Borda ${ }^{1}$, Giovanny Torres - Vidales ${ }^{2}$
}

Médico Veterinario Zootecnista, Universidad Pedagógica y Tecnológica de Colombia.

2 Veterinario Zootecnista, Profesor asistente, Programa de Medicina Veterinaria y Zootecnia. UPTC, Grupo de Investigación GIDIMEVETZ Tunja, Colombia

Email: gitovi34@hotmail.com

\begin{abstract}
Resumen
El objetivo del presente artículo es realizar una caracterización anatómica completa de las estructuras bronquiales y pulmonares del perro, que proporcione referencias claras para diferentes procedimientos de la práctica médica veterinaria. Para tal fin se utilizó un total de 8 perros criollos, con un rango de edad entre los 3 y 5 años y en buenas condiciones de salud; se realizó broncoscopia en 4 de ellos, disección fina del tejido bronquial en 3 y en un ejemplar, corte coronal de los pulmones para visualizar las ramificaciones bronquiales y su distribución dentro de los lóbulos. Los resultados demuestran que mediante las técnicas de disección fina, corte coronal y broncoscopia, se logró realizar una descripción detallada de los pulmones y el árbol bronquial de los caninos. Siendo los hallazgos más relevantes para los pulmones la existencia de un ápice, tres superficies, tres bordes. Encontrándose en el pulmón derecho tres fisuras y en el izquierdo dos fisuras. Para el tejido bronquial se encontró que el bronquio principal derecho da origen a ramas independientes para los lóbulos apical, medio y accesorio antes de entrar al lóbulo diafragmático. El bronquio principal izquierdo emite un bronquio común a las porciones craneal y caudal del lóbulo apical antes de entrar al lóbulo diafragmático. Como conclusión se platea que el presente estudio es de gran relevancia, ya que aporta conceptos claros y específicos acerca de la anatómica broncopulmonares del perro, los cuales pueden servir como herramientas que mejores las técnicas diagnósticas, médicas y quirúrgicas del tracto respiratorio bajo en caninos.
\end{abstract}

Palabras clave: Bronquio, árbol bronquial, pulmón, caninos.

\begin{abstract}
The aim of this study was perform a complete anatomical characterization of the bronchial and pulmonary structures of the dog, which provides clear references for different procedures from the veterinary medical practice. Were used for this purpose a total of 8 dogs natives, with an age range between 3 and 5 years old and in good health; bronchoscopy was performed in 4 of these, thin bronchial tissue dissection in 3 and in an individual, cut coronal lungs to display the bronchial ramifications and its distribution within the lobe. The results show that using the techniques of fine dissection and coronal
\end{abstract}


slice you achievement to carry out a detailed description of the lungs and bronchial tree for canines. Being the most relevant findings to the lungs, the existence of an apex, three surfaces, three edges. Found in the right lung and three fissures in the left two fissures. For the bronchial tissue is found that the right mainstem bronchus gives rise to separate branches for the lobes apical, middle and attachment prior to entering the diaphragmatic lobe. The left main bronchus emits a bronchus to the common portions of the cranial and caudal lobe apical before entering the diaphragmatic lobe. In conclusion this study is of great importance, since it provides concepts clear and specific about the anatomical broncho of the dog, which can serve as tools to better diagnostic techniques, medical and surgical of the lower respiratory tract in canines.

Key words: Bronchus, bronchial tree, lung, canine.

\begin{abstract}
Resumo
O objetivo deste trabalho é realizar uma caracterização anatômicas completas estruturas brônquicas e pulmonares do cão, para fornecer referências claras para os diferentes procedimentos da prática médica veterinária. Para isso, usou um total de oito cães de raça, com faixa etária entre 3 e 5 anos e com boa saúde; broncoscopia foi realizada em 4 pacientes, fina dissecção tecido brônquico em 3 e uma cópia, secção coronal dos pulmões para ver as ramificações dos brônquios e sua distribuição dentro dos lobos. Os resultados mostram que por meio de técnicas de dissecação fina, coronais e broncoscopia, foi possível fazer uma descrição pormenorizada dos pulmões e da árvore brônquica dos caninos. Sendo os achados mais importantes para os pulmões, a existência de um ápice, três superfícies, três fronteiras. Sendo no pulmão direito três rachaduras e fissuras nos dois esquerdo. Para tecido brônquico-se que o brônquio principal direito dá origem a ramos independentes lobos apicais, meio e acessórios antes de entrar no lobo diafragmático. O brônquio principal esquerdo questões brônquio comuns às porções cranial e caudal do lobo apical antes de entrar no lobo diafragmático. Em conclusão, o presente estudo é de grande importância, uma vez que fornece conceitos claros e específicos sobre o cão broncopulmonar anatômico, que podem servir como ferramentas para melhores técnicas de diagnóstico, do trato respiratório inferior médico e cirúrgico em cães.
\end{abstract}

Palavras chave: Brônquios, árvore brônquica, pulmão, cães

\section{Introducción}

La especie canina, una de las más frecuentes en la consulta veterinaria, tiene dentro de sus diversas patologías algunas que cursan con alteraciones respiratorias que requieren adecuadas técnicas diagnósticas, procedimientos médicos o quirúrgicos específicos del sistema respiratorio y terapias como la fisioterapia del tórax; haciéndose indispensable el completo conocimiento de las características anatómicas broncopulmonares del perro.

La anatomía broncopulmonar del perro ha sido descrita por diversos autores como Amis y McKiernan 1986, quienes realizaron una identificación sistemática de la anatomía bronquial basándose en la observación broncoscópica; igualmente Alados et al 1996 y Canals et al 1998, trabajaron en una caracterización de la geometría fractal del árbol bronquial en mamíferos incluyendo caninos. Se encuentran además descripciones anatómicas en diferentes textos del área médica, pero no se cuenta con información que relacione la anatomía de las estructuras bronquiales con los lóbulos pulmonares, y debido a que se dificulta la obtención detallada de información relacionada con la disposición bronquial se hace necesaria la búsqueda de la misma mediante técnicas como la disección fina, la obtención de moldes bronquiales y la visualización directa mediante broncoscopio.
Son pocos los estudios acerca de la estructura anatómica del árbol bronquial canino en relación a su distribución respecto a los lóbulos pulmonares por lo cual, también han sido subutilizadas técnicas de ayuda en el tratamiento de las enfermedades broncopulmonares como lo es la terapia respiratoria o fisioterapia del tórax. No han sido publicados estudios al respecto en el país, y la información reportada en los textos de anatomía fue obtenida hace más de 20 años, por lo cual es de gran importancia dar un primer paso en el estudio del sistema respiratorio que suscite el interés de investigadores y clínicos en la implementación de nuevas herramientas para el manejo de pacientes con alteraciones de este sistema.

Se busca obtener una información clara y específica acerca de las características anatómicas broncopulmonares en caninos que permita orientar de manera exacta y definida para la especie, procedimientos como la broncoscopia, el lavado bronquial, la interpretación radiográfica simple y la broncografía, del mismo modo que sirva como referencia en procedimientos como la extracción de cuerpos extraños en árbol bronquial o el tejido pulmonar, entre otros. El conocimiento de la distribución bronquial y su concordancia con los lóbulos pulmonares permite diseñar protocolos de actuación en diversos procedimientos como los mencionados anteriormente y aplicar de forma explícita nuevos recursos terapéuticos como la terapia respiratoria y 
dentro de ésta el drenaje bronquial basándose en segmentos específicos a drenar, por medio de posiciones apropiadas.

\section{Materiales y métodos}

El estudio se realizó en las instalaciones de la Clínica Veterinaria de Pequeños y Grandes Animales de la Universidad Pedagógica y Tecnológica de Colombia, sede Tunja. El manejo de los animales se realizó de acuerdo a lo estipulado por las Leyes 84 de 1989 y 576 de 2000 que reglamentan para Colombia la utilización de animales en experimentos e investigación.

Población. Se utilizaron 8 perros de raza criolla, 4 hembras y 4 machos, con un rango de edad entre los 3 a 5 años, con un peso que osciló entre 20 y $25 \mathrm{Kg}$ y en buenas condiciones de salud, la cual fue constatada mediante examen clínico completo. Se emplearon 4 caninos para broncoscopia, 3 para realizar disección fina y uno para corte coronal pulmonar. Los animales seleccionados estaban destinados a eutanasia de acuerdo con lo establecido por el programa de Zoonosis de la Secretaría de Protección Social de Tunja.

Broncoscopia. Para la realización de broncoscopia, se utilizó el siguiente protocolo de anestesia fija: en la preanestesia se administró Atropina 0,02 mg/kg S.C. y Acepromacina $0,1 \mathrm{mg} / \mathrm{kg}$ I.M.; a los 30 minutos se administró para Inducción un bolo con un tercio de la dosis total de Xilacina 0,5 mg/kg I.V. y Ketamina 8 mg/ kg I.V cargadas en la misma jeringa. Posteriormente se administraron los 2/3 restantes para profundización de la misma forma. La broncoscopia fue realizada con un Fibropanendoscopio Olympus, modelo GIF-E3, de 1 metro de longitud y campo visual de $120^{\circ}$. Con el animal en decúbito dorsal, con el cuello extendido, se introdujo el broncoscopio en forma directa a través de la laringe, hasta llegar a la tráquea y visualizar la carina, luego de lo cual se examinó cada bronquio y sus ramificaciones en forma sistemática, hasta donde el diámetro de la vía respiratoria permitió el avance del endoscopio. Se utilizó una nomenclatura para los bronquios mayores y sus subdivisiones para facilitar la descripción de los hallazgos broncoscópicos, como lo muestra la Tabla 1.

Disección fina. En los caninos destinados a este procedimiento, se realizó eutanasia mediante inyección intravenosa de Eutanex ${ }^{\circledR}$, a dosis de $1 \mathrm{~mL}$ por cada 5 $\mathrm{kg}$ de peso vivo. Posteriormente, con el paciente en decúbito dorsal se incidió piel y tejido subcutáneo en la línea media del cuello desde el cartílago cricoides hasta el esternón y directamente sobre el esternón desde el manubrio hasta la apófisis xifoides, se con- tinuó incidiendo las inserciones musculares sobre la línea media para exponer la tráquea y el esternón luego de lo cual se realizó osteotomía cuidando de no dañar las estructuras del tórax. Luego se retiraron los órganos del sistema respiratorio partiendo de la tráquea, éstos se dejaron sumergidos durante 48 horas en formol al $10 \%$. Posteriormente y luego de enjuagar con abundante agua, se procedió a retirar el parénquima pulmonar mediante el uso de pinzas de disección, tijeras y bisturí, hasta dejar expuesto el árbol traqueobronquial, el cual nuevamente se deja en inmersión en formol al 10\% por un tiempo de 72 horas. En uno de los ejemplares, no se realizó disección fina del sistema bronquial, sino un corte coronal de los pulmones para visualizar las ramificaciones bronquiales y su distribución dentro de los lóbulos.

\section{Resultados}

Broncoscopia. Mediante esta técnica fue posible evidenciar que las características anatómicas bronquiales observadas por este método no variaron entre los cuatro animales estudiados, pudiéndose observar las ramificaciones bronquiales en forma completa hasta bronquios lobulares. Se observó la entrada de los bronquios segmentarios, pero no se tuvo pleno acceso al interior de estas ramificaciones (Figura 1).

En el lóbulo medio derecho, se visualizaron las primeras ramificaciones rostrales y caudales (R1 y C1). Como característica importante, se observó que el ligamento dorsal de la tráquea, debido a su disposición define más la entrada hacia el bronquio principal izquierdo, que al derecho (Figura 2).

Disección fina. En los tres especímenes se logró obtener un árbol bronquial completo, previa fijación de éste en formol al 10\% durante 48 horas (Figura 3). Así mismo, el corte coronal de los pulmones en un espécimen permitió comparar la distribución bronquial de la disección fina con la obtenida en cada lóbulo (Figura 4).

Caracterización anatómica broncopulmonar del perro. A partir de la información que se obtuvo mediante la broncoscopia, la disección fina y el corte coronal se recopilaron los datos necesarios para realizar una caracterización broncopulmonar, soportada en la visualización directa, las fotografías obtenidas de los procedimientos, los bronquios y pulmones disectados conservados en formol.

Morfología externa de los pulmones. Los pulmones son órganos respiratorios pares, derecho e izquierdo, ubicados en la cavidad torácica ocupando la mayor parte de ésta; los pulmones observados fueron de co- 
Tabla No. 1 Nomenclatura para bronquios mayores y sus subdivisiones para la descripción de los hallazgos broncoscópicos

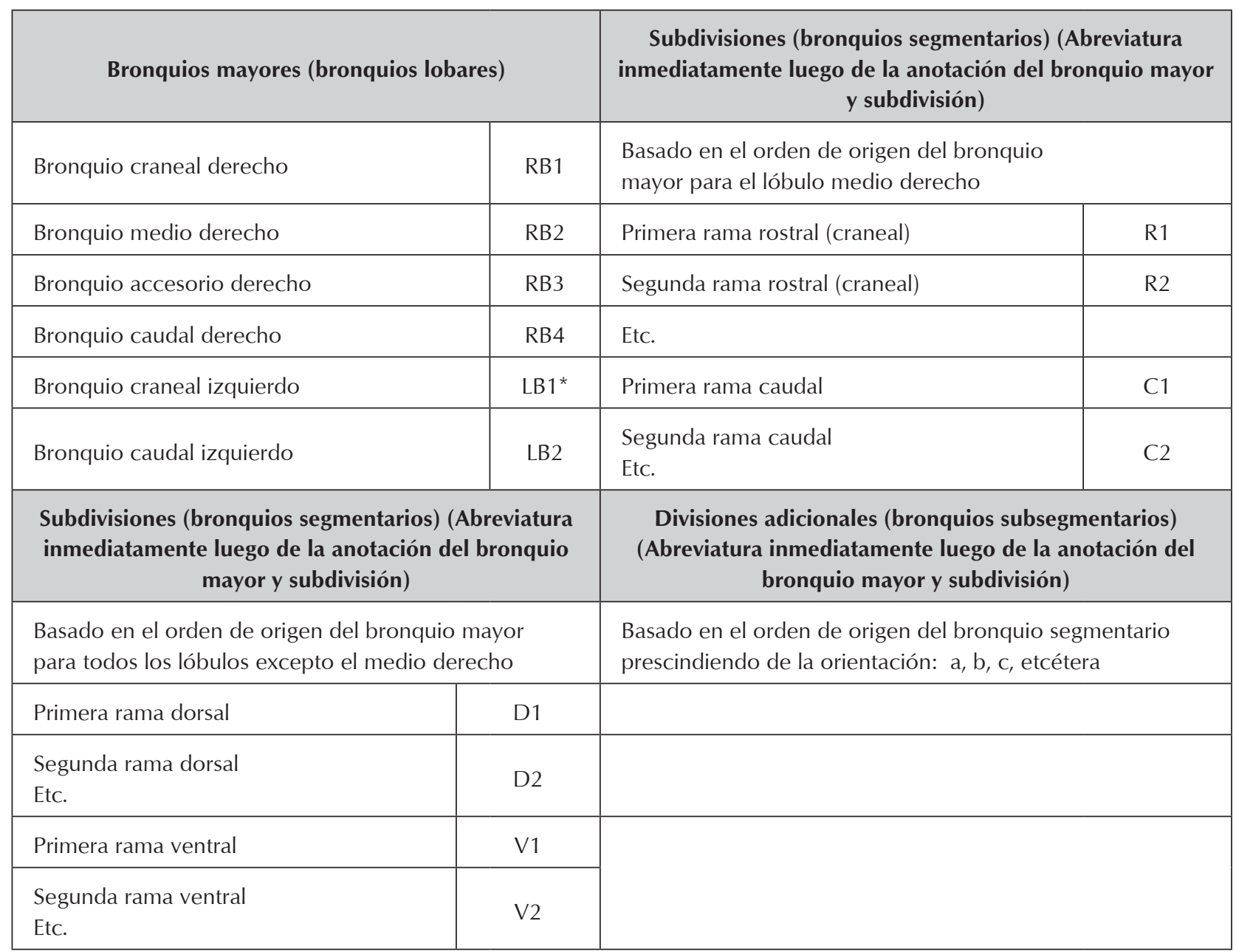

* El bronquio hacia la parte caudal del lóbulo craneal izquierdo se identifica como LB1V1 (Amis and McKiernan, 1986).

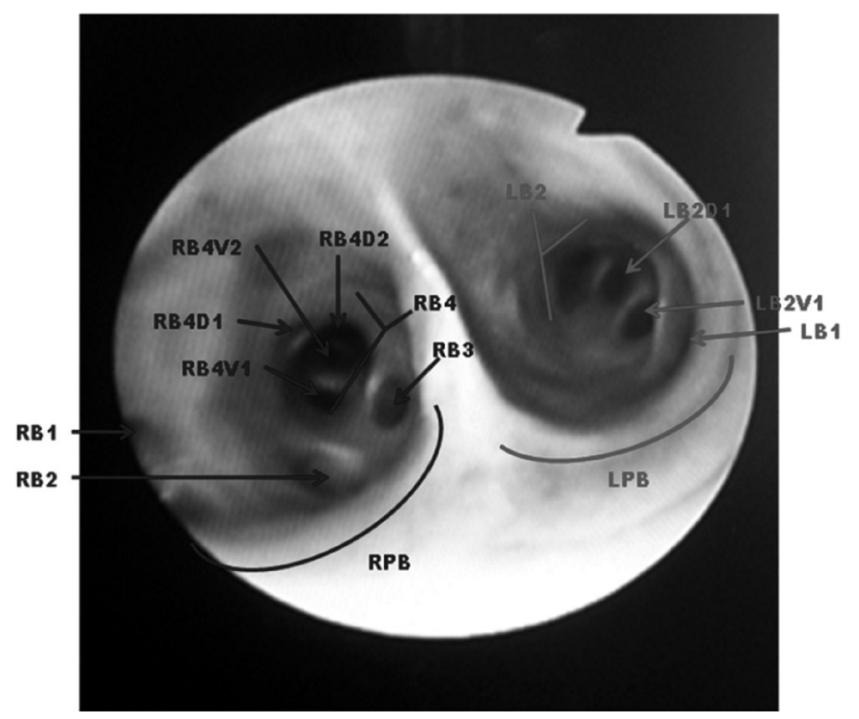

Figura 1. Vista broncoscópica de los bronquios principales y sus ramificaciones. Según nomenclatura propuesta en tabla 1. 


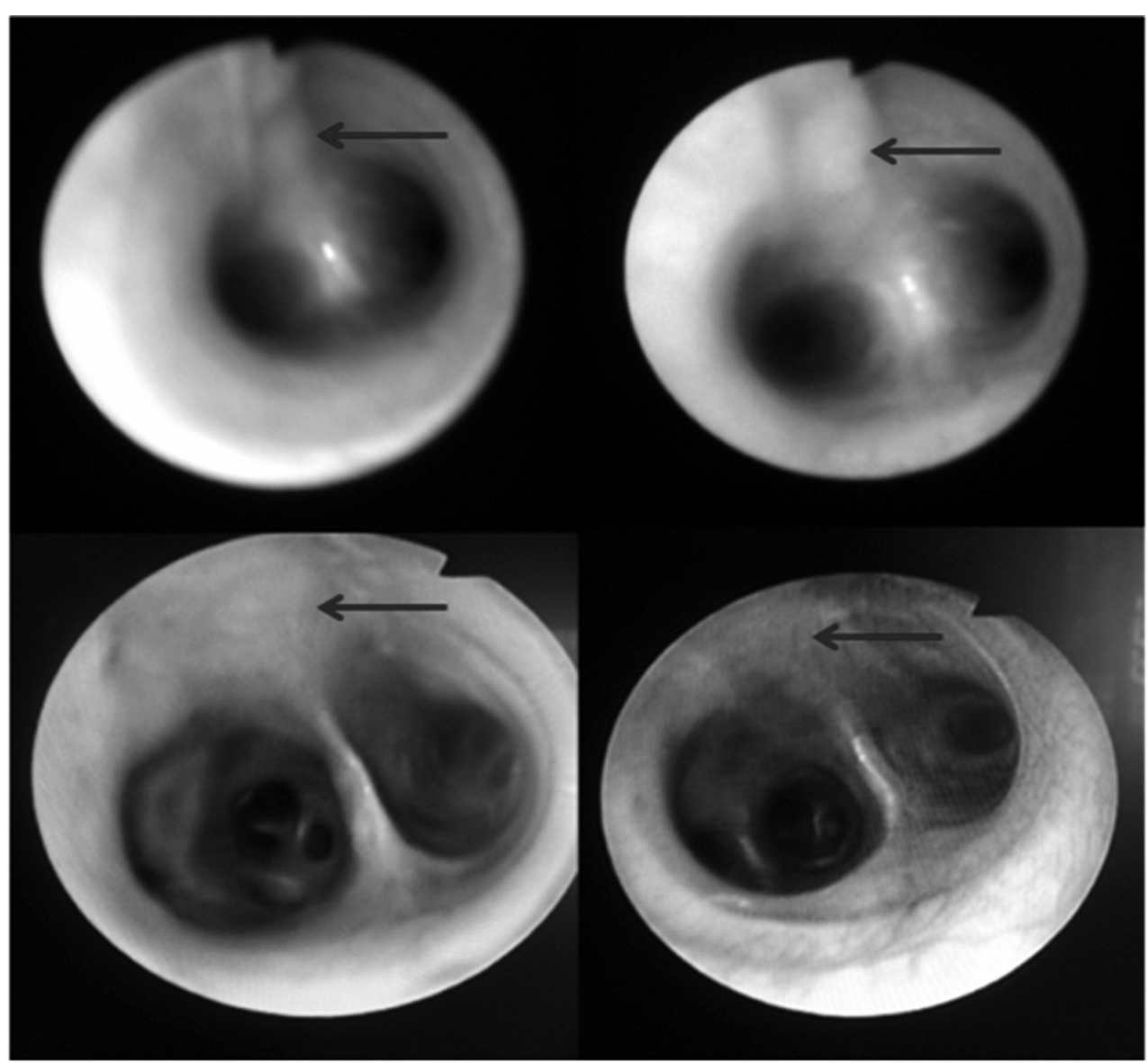

Figura 2. Visualización broncoscópica de la carina de los cuatro animales estudiados mediante esta técnica, señalando el ligamento dorsal de la tráquea que define el origen del bronquio principal izquierdo en forma más acentuada que el derecho.

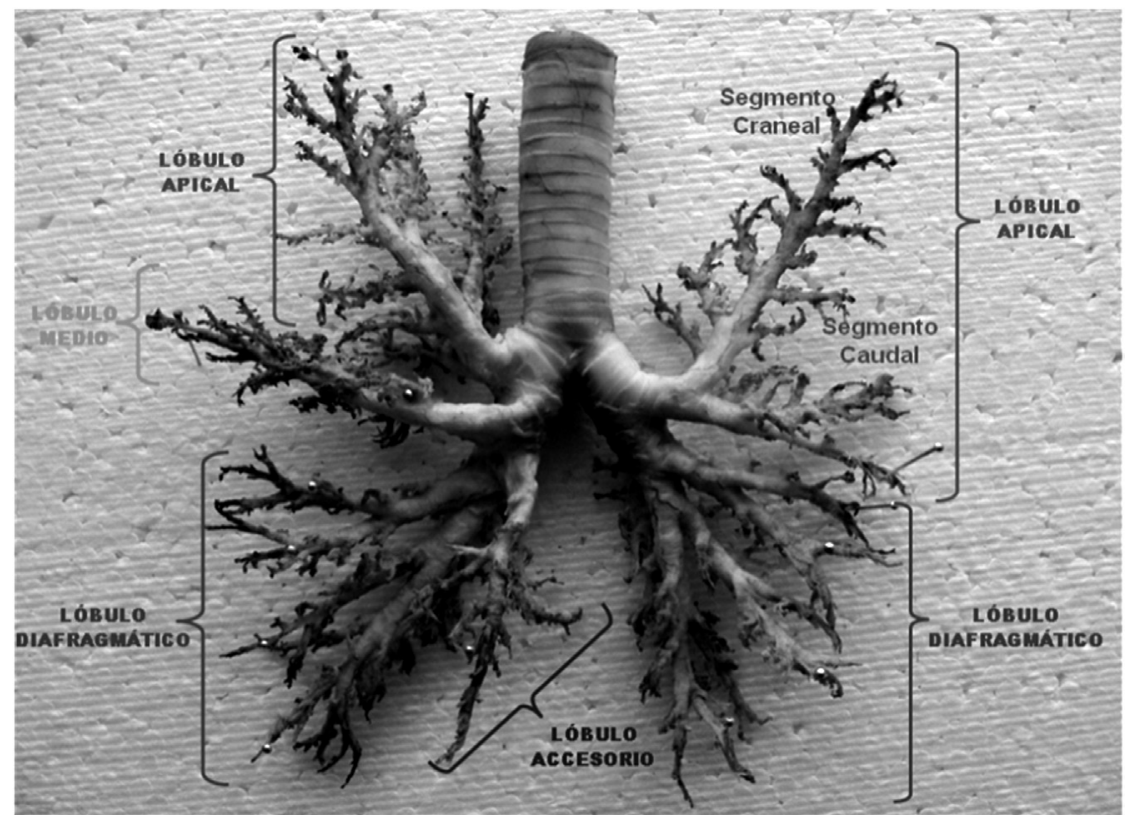

Figura 3. Árbol bronquial con la distribución a cada lóbulo, Técnica Disección Fina. Vista ventrodorsal. 


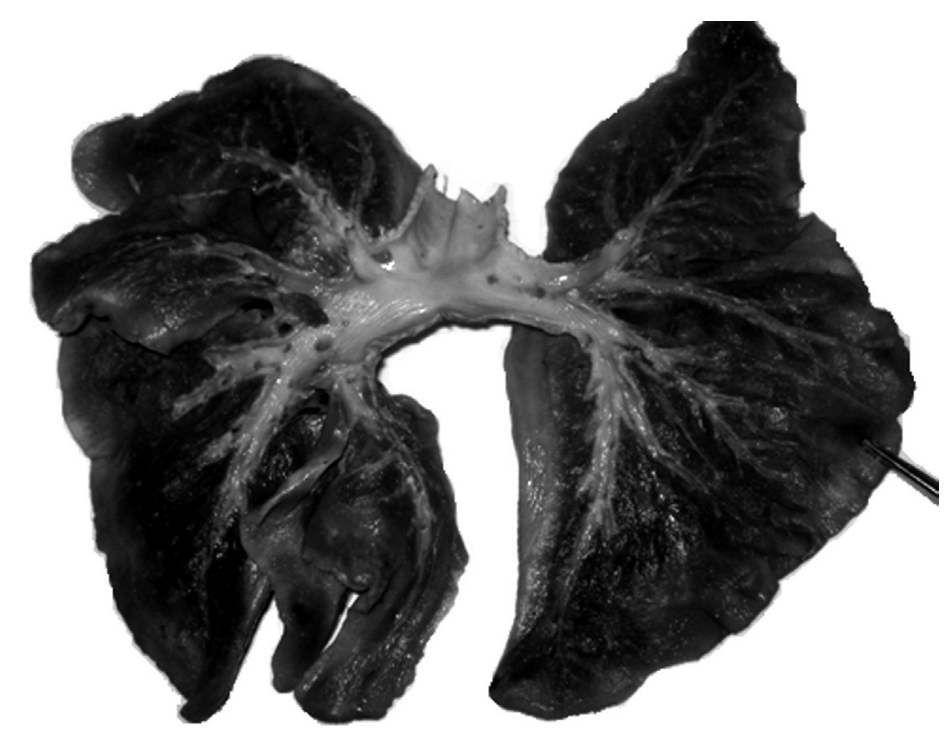

Figura 4. Corte coronal del pulmón, vista ventrodorsal, se definen claramente cada una de las divisiones de los bronquios intrapulmonares

lor rosado pálido ajustándose a las características del pulmón sano, con superficie brillante y lisa, bordes irregulares y forma triangular (Figura 5). En cada pulmón se identificó un ápice, tres superficies: costal, medial y diafragmática, y tres bordes: dorsal, ventral y caudal. El ápice, con forma redondeada, constituye lo que se podría llamar "la punta más aguda del triángulo".

La superficie costal, de forma convexa, corresponde a la cara lateral o externa del pulmón y estaba limitada por los bordes dorsal, ventral y caudal; el lóbulo accesorio no fue visible en esta superficie. La superficie medial la constituyen dos porciones dorsal y ventral; la porción dorsal está en contacto con la columna vertebral y la ventral con el mediastino amoldándose a su contenido, de lo cual se obtiene la impresión cardiaca, dorsal y caudal a la cual se encontró el hilio pulmonar.

En la superficie medial del pulmón derecho sobresalió el lóbulo accesorio, el cual se ubica en la porción caudal al corazón rodeando la vena cava caudal. La superficie diafragmática, tiene forma cóncava, en el pulmón derecho se mostró más amplia que en el izquierdo, formada por los lóbulos diafragmático, medio

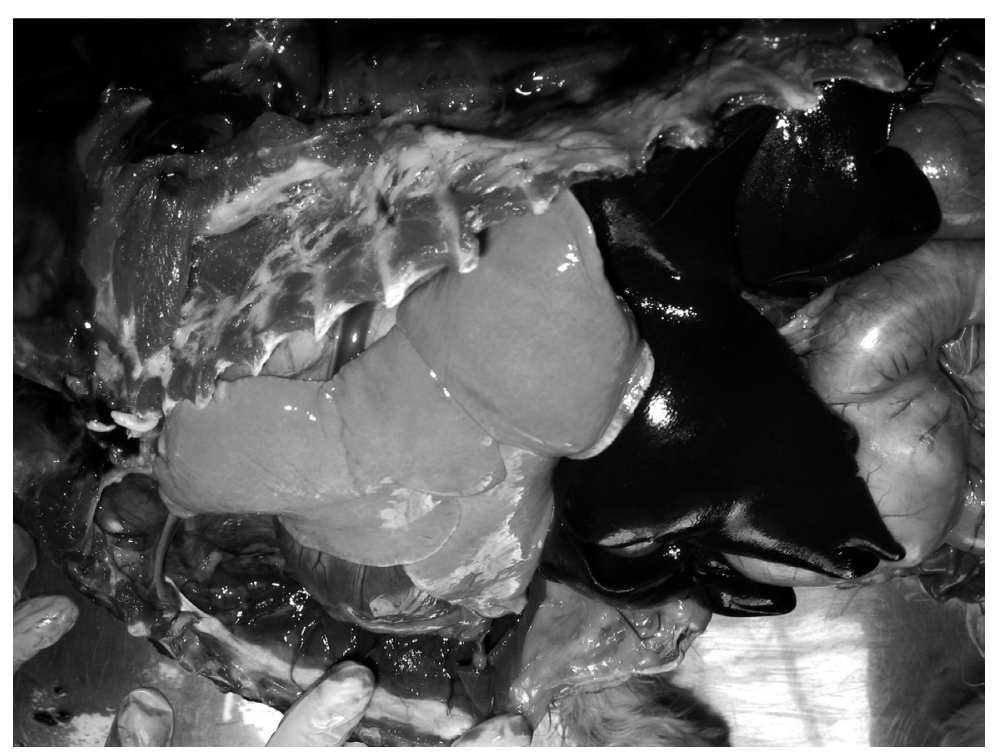

Figura 5. Vista de pulmón izquierdo, se destaca posición anatómica, morfología, color y textura. Propios de un órgano sano. 
y accesorio, mientras que en el pulmón izquierdo solamente la formaban el lóbulo diafragmático y la porción caudal del lóbulo apical.

El borde dorsal, de forma redondeada, está en contacto con el cuerpo de las vértebras torácicas y formado por los lóbulos apical y diafragmático de cada pulmón. El borde ventral con su terminación fina, se extiende desde el ápice hasta el extremo ventral del lóbulo medio en el pulmón derecho y, desde el ápice hasta el extremo ventral de la porción caudal de lóbulo apical en el pulmón izquierdo; hacia la parte media en ambos pulmones en el borde ventral se observó la escotadura cardiaca, la cual era más acentuada en el pulmón derecho que en el izquierdo. El borde caudal rodea la superficie diafragmática de cada pulmón.

En cada pulmón se observaron fisuras profundas que los dividen en lóbulos. En el pulmón derecho se observaron cuatro lóbulos: apical, medio, diafragmático y accesorio, y en el izquierdo dos lóbulos: apical y diafragmático; el lóbulo apical del pulmón izquierdo, dividido en dos porciones craneal y caudal. Las fisuras halladas en el pulmón derecho son tres: fisura caudal separando el lóbulo diafragmático del apical y medio; fisura craneal separando los lóbulos apical y medio; fisura paramedial separando los lóbulos diafragmático y accesorio. En el pulmón izquierdo dos fisuras: fisura craneal separando los segmentos o porciones craneal y caudal del lóbulo apical; fisura caudal separando el lóbulo apical del diafragmático (Figura 6).

Morfología bronquial. La tráquea se bifurca en bronquios principales derecho e izquierdo, dorsal a la aurícula izquierda del corazón. El bronquio principal derecho da origen a ramas independientes para los lóbulos apical, medio y accesorio antes de entrar al lóbulo diafragmático. El bronquio principal izquierdo emite un bronquio común a las porciones craneal y caudal del lóbulo apical antes de entrar al lóbulo diafragmático. Así, cada bronquio principal, se continúa como bronquio lobular del lóbulo diafragmático, formando una curva cóncava dorsomedialmente y reduciendo gradualmente su calibre (Figura 3).

En el lado derecho, el bronquio del lóbulo apical se origina a partir de la superficie craneolateral, el bronquio del lóbulo medio de la superficie ventral, y el bronquio del lóbulo accesorio de la superficie ventromedial del bronquio principal. En el lado izquierdo el bronquio del lóbulo apical se origina a partir de una posición levemente más ventral y caudal que el bronquio del lóbulo apical derecho y dividiéndose en dos porciones craneal y caudal después de una corta distancia. Cada bronquio lobular atraviesa su respectivo lóbulo dirigiéndose desde el hilio a un punto terminal que fue constante en los animales estudiados (Figura 3).

Los bronquios lobulares presentan ramificaciones COnocidas como bronquios segmentarios, las cuales a su vez se subdividen en subsegmentarios. El número total de bronquios segmentarios y subsegmentarios variaron de lóbulo a lóbulo y de un pulmón a otro. En los lóbulos apicales, diafragmáticos y accesorio, la serie de bronquios principales tenían origen dorsal y ventral, pudiéndose designar por las letras $\mathrm{D}$ y $\mathrm{V}$ respectivamente. En el lóbulo medio derecho, las series princi-

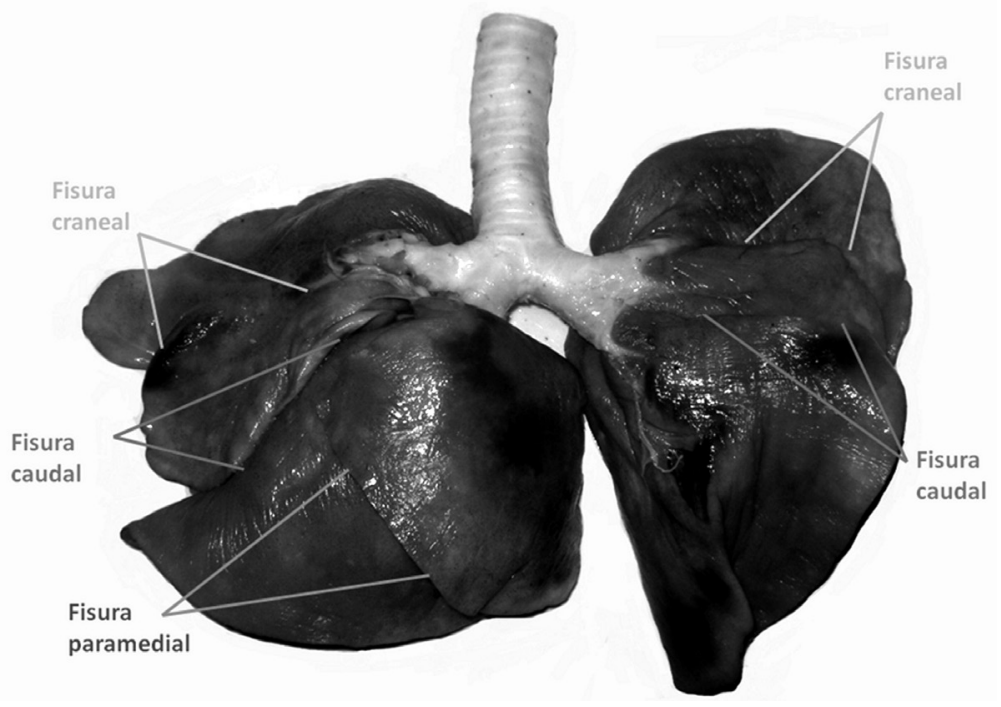

Figura 6. Fisuras pulmonares, vista ventrodorsal. 
pales se dirigen craneal y caudalmente, pudiéndose señalar por las letras R y C (rostral y caudal).

Bronquios lobulares. Los bronquios lobulares excepto en el lóbulo apical izquierdo mostraron una porción intrapulmonar larga y una extrapulmonar corta. Las porciones craneal y caudal del lóbulo apical izquierdo tienen su origen en un bronquio lobular común y no mostraron porciones extrapulmonares. El bronquio común era totalmente extrapulmonar (Figura 4).

El bronquio lobular del lóbulo apical derecho forma una curva en forma de $\mathrm{C}$ entre su origen y terminación. Se origina en frente o levemente craneal al nivel del ángulo traqueal Ilegando hasta el extremo distal del lóbulo, situado en el borde ventral del pulmón en el ápice.

El bronquio lobular del lóbulo medio derecho se dirige ventrolateralmente desde su origen, atravesando el lóbulo para alcanzar el extremo distal de éste en un punto cercano a la unión del borde ventral con el caudal.

El bronquio lobular del lóbulo accesorio se origina antes de que el bronquio lobular ingresara en el lóbulo diafragmático. Su parte extrapulmonar corta tenía dirección caudoventral y medial, atravesando luego el lóbulo oblicuamente, dirigiéndose hacia el extremo distal del lóbulo.

El bronquio lobular del lóbulo diafragmático derecho puede decirse que es la continuación del bronquio principal derecho, el cual atravesaba el hilio del lóbulo dirigiéndose hacia el extremo distal, que es la porción más caudal del pulmón.

El bronquio común de las porciones craneal y caudal del lóbulo apical izquierdo tiene su origen a un nivel caudal al ángulo de la bifurcación de la tráquea, continuando en dirección ventrolateral para en un corto trayecto dividirse en las porciones respectivas.

En el bronquio lobular de la porción craneal del lóbulo apical izquierdo se observó que éste gira cranealmente para atravesar el lóbulo hasta su extremo distal en un punto medio entre los bordes dorsal y ventral, que coincide con el ápice del pulmón izquierdo.

El bronquio lobular de la porción caudal de lóbulo apical izquierdo se dirige ventrolateralmente desde su origen, atravesando el lóbulo para alcanzar el extremo distal de éste ubicado en un punto medio entre la unión del borde ventral con el caudal.

Del bronquio lobular del lóbulo diafragmático izquierdo al igual que en el derecho, puede decirse que es la continuación del bronquio principal izquierdo, que atraviesa el hilio del lóbulo dirigiéndose hacia el extremo distal, que es la porción más caudal del pulmón.

\section{Discusión}

Las técnicas de broncoscopia, disección fina y corte coronal son apropiadas para la caracterización anatómica del árbol bronquial y la división lobular del pulmón del perro, porque permiten la visualización directa de estas estructuras.

En la evaluación externa de los pulmones, se comprobó que el derecho está compuesto por cuatro lóbulos: apical, medio, diafragmático y accesorio. Separados entre sí por tres fisuras y el pulmón izquierdo con dos lóbulos el apical y el diafragmático y dos fisuras. Estos hallazgos son disimiles a los reportados por Ishaq en 1980, quien indica que el pulmón izquierdo cuenta con tres lóbulos, pero concuerda con lo reportado por Nikakuki en 1994, quien describe la presencia de dos lóbulos para el pulmón izquierdo. Así mismo Nakakuki en 1993, en un trabajo realizado con 5 individuos de la especie equina encontró que esta cuanta con tres lóbulos en el pulmón derecho y dos en el pulmón izquierdo. Lo que pone de manifiesto una clara diferencia con lo reportado por nuestro estudio en la especie canina.

El hallazgo más relevante en la visualización broncoscópica se relaciona con la mayor definición del origen del bronquio principal izquierdo (figura 2), proporcionada por el ligamento dorsal de la tráquea. Lo cual no ha sido reportado por autores que han realizado trabajos similares (Nakakuki, 1994; Horsfield et al., 1982) y que puede sugerir mayor riesgo de broncoaspiración hacia los segmentos broncopulmonares del lado izquierdo, como lo referencian Silverstein D. y Drobatz K, 2005, de la auscultación torácica con algunos trastornos respiratorios, en donde se afirma que los ruidos accesorios que se escuchan en la zona craneal del pulmón izquierdo pueden ser un indicio de neumonía por aspiración (Etinger and Felman, 2007).

Tomando como base la disección fina, se sugiere que la disposición bronquial en los lóbulos pulmonares difiere de las figuras encontradas en textos de referencia como la anatomía de los animales domésticos de Sisson, S. y Grossman J 1998, quienes en la figura 5217 Arborización bronquial de los pulmones del perro; vista dorsal, no identifican una bifurcación clara de la tráquea, lo cual hace parecer como si el bronquio apical derecho e izquierdo se originaran en la tráquea, craneal a la bifurcación y no a partir del bronquio principal luego de la bifurcación, echo que si documenta 
Ishaq en un estudio realizado en 1980 utilizando 37 pares de pulmones de caninos.

Del mismo modo Köning H E, y Liebich H G. (2011); Ettinger (2007), Adams (2004), así como Sisson S, y Grossman, JD. (1998) en sus diagramas de la anatomía bronquial normal en perros ilustran esquemas en los cuales se muestra el origen del bronquio lobular apical izquierdo no como un bronquio común que se bifurca en bronquio segmentario craneal y caudal, sino como dos bronquios lobulares que se originan a partir del bronquio principal.

Finalmente se recomienda la realización de investigaciones que tomen como punto de partida la anatomía broncopulmonar del perro, para determinar aspectos como el riesgo de broncoaspiración y las modificaciones posturales para procedimientos como el lavado broncoalveolar o la extracción de cuerpos extraños de segmentos broncopulmonares específicos.

\section{Agradecimientos}

El presente trabajo se desarrolló dentro del cronograma de actividades de investigación del grupo GIDIMEVTZ. Los autores agradecen a las directivas de la Clínica Veterinaria de la Universidad Pedagógica y Tecnológica de Colombia por el préstamo de los equipos e instalaciones para el desarrollo de las actividades.

\section{Referencias}

Adams RD. Canine Anatomy, A systematic study, Fourth Edition. lowa state press, USA 2004. p. 197-201.

Alados CL, Escos JM, Emlen JM. Fractal structure of sequential behaviour patterns: an indicator of stress. Anim Behav. 1996; 51:437-43
Canals M, Olivares R, Labra F, Caputo L, Rivera A, Novoa F. Caracterización de la geometría fractal del árbol bronquial en mamíferos. Rev Chil Anat. 1998; 16(2): 237-244.

Ettinger SJ, Feldman EC. Tratado de Medicina Interna Veterinaria. Elsevier. Sexta Edición 2007. Volumen II, p. 1206-1288.

Gil J. Models of lung disease: Microscopy and structural methods. Vol. 47. New York: Marcel Dekker; 1990.

Horsfield K, Kemp W, Phillips S. An asymmetrical model of airways of the dog lung. J Appl Physiol, 1982; 52(1): 21-26.

Horst E, Konig HE, Liebich HG. Anatomía de los animales domésticos Tomo dos, segunda edición corregida y Ampliada, editorial médica panamericana, 2008 madrid pag: 97-102.

Ishaq M. A morphological study of the lungs and bronchial tree of the dog: with a suggested system of nomenclature for bronchi. J Anat, 1980; 131(4): 589-610.

Köning HE, Liebich HG. Anatomía de los animales domésticos, Texto y atlas en color. Editorial Médica Panamericana, Bogotá Colombia, 2011. Tomo 2, p. 98.

Nakakuki S. The bronchial tree and lobular division of the dog lung. J Vet Med Sci. 1994; 56(3): 455-458.

Nakakuki S. The bronchial tree, lobular division and vessels of the japonese deer (cervus Nippon) lung. J Vet Med Sci. 1993; 55(3): 443-447.

Nakakuki s. The bronchial tree and lobular division of the horse lung. J Vet Med Sci. 1993; 55(3): 435-438.

Pump KK. The morphology of the finer branches of the bronchial tree of the human lung. Dis Chest. 1964; 46: 379-398.

Silverstein DC, Drobatz KJ.: "Clinical evaluation of the respiratory tract" "Textbook of Veterinary Internal Medicine: Diseases of the Dog and Cat," 6th Ed. Page: 1206-1217, Ettinger SJ and Feldman EC eds. (eds.). W.B. Saunders, Philadelphia , 2005.

Sisson S, Grossman, JD. Anatomía de los animales domésticos $5^{\mathfrak{a}}$ ed. Vol 2. España: Masson; 1998.p. 1725. 